\title{
Causes des variations phénotypiques de la mucoviscidose
}

\author{
Garry R. Cutting \\ Institute of Genetic Medicine, Johns Hopkins Hospital, Baltimore, Md., États-Unis
}

\section{Mots-clés}

Gènes modificateurs • Facteurs environnementaux • Gène du CFTR

\section{Résumé}

La mucoviscidose est due à une anomalie fonctionnelle du gène régulateur de la conductance transmembranaire de la mucoviscidose (CFTR). L'évolution de la mucoviscidose est très variables, certains sujets décédant de cette maladie au cours de leur première décennie tandis que d'autres atteignent leurs quatrième et cinquième décennies. Les atteintes viscérales et les complications diffèrent également entre les patients. Certaine des variations phénotypiques de la mucoviscidose peuvent être attribuées à la nature de l'anomalie du gène du CFTR. Le génotype CFTR détermine principalement le degré de dysfonction pancréatique exocrine et est corrélé au degré d'anomalie de la concentration du chlorure dans la sueur et de malformation de l'appareil génital masculin. Des facteurs indépendants du CFTR sont cependant responsable de variations de l'atteinte respiratoire, principale cause de morbidité et de mortalité dans la mucoviscidose. Ces dernières années, des gènes modificateurs influençant la sévérité de l'atteinte respiratoire ont été identifiés chez des patients atteints de mucoviscidose. Des facteurs non génétiques tels que le statut socio-économique et le tabagisme passif influencent également l'évolution respiratoire. Des connaissances plus complètes des causes des variations entre les patients atteints de mucoviscidose sont en cours d'acquisition, facilitant le développement de nouvelles mesures pronostiques et de traitements innovants.

Copyright @ 2006 Nestec Ltd., Vevey/S. Karger AG, Basel (c) 2006 Nestec Ltd., Vevey/S. Karger AG, Basel 0250-9644/06/0643-0111\$23.50/0

ax +41 613061234

E-Mail karger@karger.ch

www.karger.com
Accessible en ligne à: www.karger.com/anf

\section{Introduction}

La mucoviscidose, maladie monogénique qui atteint 60000 personnes au plan mondial, est due à des anomalies du gène régulateur de la conductance transmembranaire de la mucoviscidose (CFTR). Elle atteint principalement des sujets d'origine caucasienne européenne avec une incidence de 1/2 000 à 1/4 000 [1]. Elle est beaucoup moins fréquente dans les populations d'origine africaine et asiatique. La fréquence de la mucoviscidose est plus élevée dans certains groupes caucasiens que dans d'autres, sans doute en raison d'un effet fondateur [2, 3]. La cause de la fréquence élevée de porteurs de mutations à l'origine de la mucoviscidose chez les Caucasiens (1 sur 30) n'est pas connue, bien qu'un avantage pour l'hétérozygote soit l'explication préférée [1]. L'implication de tout mécanisme doit prendre en compte le fait que, chez les Caucasiens, la mucoviscidose est due à la présence d'une mutation fréquente qui est absente dans d'autres groupes ethniques, sauf dans des cas imputables à un métissage. De ce fait, l'explication de la fréquence élevée de cette mutation ( $\Delta \mathrm{F} 508$; voir plus bas) expliquera sans doute également la prévalence élevée de la mucoviscidose chez les Caucasiens. L'étiologie de la mucoviscidose est désormais connue, et la question actuelle est donc de déterminer pourquoi l'évolution de cette maladie diffère en fonction des patients. La revue qui suit présente une synthèse des connaissances actuelles sur le rôle des variations du gène du CFTR ainsi que des données émergentes sur l'importance de facteurs indépendants de ce gène dans la détermination de la sévérité d'une mucoviscidose. 


\section{Variations phénotypiques de la mucoviscidose}

A l'époque de la première description de la mucoviscidose en tant qu'entité distincte dans les années 1940, la plupart des patients atteints de cette maladie en décédaient avant leur premier anniversaire en raison d'une malnutrition due à la dysfonction du pancréas exocrine. Le traitement de l'insuffisance pancréatique exocrine, particulièrement le développement du traitement enzymatique substitutif, a permis aux patients de survivre au-delà de leur première décennie. La survie médiane a progressivement augmenté depuis les années 1940, et l'espérance de vie médiane des patients atteints d'une mucoviscidose est actuellement de 36,2 ans aux États-Unis. Malgré cet accroissement impressionnant, l'âge du décès continue à varier fortement en fonction des patients. Certains décèdent avant leur $10^{\text {ème }}$ anniversaire, tandis que d'autres atteignent la cinquantaine ou la soixantaine. L'atteinte respiratoire est actuellement la principale cause de morbidité et de mortalité dans la mucoviscidose et est actuellement responsable de $90 \%$ des décès dus à cette maladie [4]. La sévérité de cette atteinte varie considérablement en fonction des patients. Par exemple, certains d'entre eux présentent un profil obstructif au cours des premières années de leur vie, compliqué d'infections dues à Pseudomonas aeruginosa, à des champignons et, plus tard dans la vie, à des mycobactéries atypiques. D’autres présentent relativement peu de troubles respiratoires jusqu'à leur troisième décennie, puis un épisode d'hémoptysie survient et est suivi d'une dégradation rapide aboutissant à la nécessité d'une transplantation pulmonaire au bout de quelques années. La plupart des patients atteints d'une mucoviscidose souffrent également d'une rhinosinusite chronique et un sous-groupe présente une polypose nasale.

Des anomalies pancréatiques sont présentes chez la quasi-totalité des patients mucoviscidosiques. La plupart sont atteints d'une insuffisance exocrine sévère nécessitant un traitement enzymatique substitutif afin de permettre une absorption suffisante des lipides et glucides et d'éviter des carences vitaminiques. L'absorption des lipides et des glucides est satisfaisante sans traitement enzymatique dans une faible proportion (5-10\%) de patients, qualifiés de suffisants pancréatiques. Chez ces patients, l'évolution de la maladie est généralement moins sévère avec une atteinte respiratoire moins marquée, des anomalies plus discrètes de la concentration de chlorure dans la sueur et moins de complications digestives [5]. Certains présentent toutefois des troubles respiratoires sévères nécessitant une transplantation pulmonaire. La mu- coviscidose affecte également le pancréas endocrine. Tandis qu'une majorité de patients atteignait l'âge adulte, des études ont montré qu'une intolérance au glucose survenait dans une importante proportion d'entre eux et qu'un diagnostic de diabète était posé chez $30 \%$ vers l'âge de 35 ans [6]. La survenue d'un diabète a été associée à une atteinte respiratoire plus sévère et à une réduction de la longévité [7].

D'autres manifestations digestives peuvent survenir au cours de la mucoviscidose comme une hépatopathie et une occlusion intestinale. La plupart des patients présentent un certain degré d'atteinte hépatobiliaire à l'autopsie, et une hépatopathie sévère, avec cirrhose, hypertension portale et varices œsophagienne, survient chez environ $5 \%$. Lapparition de ces troubles est habituellement associée à une réduction de la survie; environ $2 \%$ des patients décèdent de complications d'une atteinte hépatique [4]. Une occlusion intestinale en période néonatale, appelée iléus méconial (IM), est une complication bien reconnue, qui affecte environ $15 \%$ des patients mucoviscidosiques [4]. Grâce aux techniques modernes de chirurgie pédiatrique, elle n'est fatale que dans des cas rarissimes. Dans certaines situations, l'occlusion peut être levée par un lavement à la gastrograffine. Un syndrome d'occlusion intestinale distale (OID) affecte des enfants plus âgés et des adultes atteints d'une mucoviscidose. Selon des estimations, 7 à $40 \%$ des patients mucoviscidosiques présentent un épisode d'OID au cours de leur vie [9]. L'IM et les OID ont des caractéristiques cliniques similaires, et taux plus élevé d'OID a été observé chez des patients ayant présenté un IM, ce qui suggère que ces deux complications partagent une étiologie commune.

Laugmentation de la concentration de sodium et de chlorure dans la sueur est une caractéristique biochimique spécifique de la mucoviscidose. L'anomalie de la concentration du chlorure est plus spécifique de la mucoviscidose et utilisée comme critère diagnostique depuis plus de quarante ans. Les concentrations de chlorure dans la sueur varient parmi les sujets en bonne santé et les patients mucoviscidosiques. Ces concentrations peuvent être de 40 à $60 \mathrm{mmol} / \mathrm{l} \mathrm{chez}$ certains adultes en bonne santé mais, chez un nourrisson ou un enfant, des valeurs de cet ordre sont compatibles avec un diagnostic de mucoviscidose. La concentration du chlorure dans la sueur est supérieure à $60 \mathrm{mmol} / \mathrm{l}$ (moyenne : environ $105 \mathrm{mmol} /$ 1) chez la plupart des patients atteints d'une mucoviscidose. Des anomalies de la concentration d'ions dans la sueur peut aboutir à une perte excessive de $\mathrm{NaCl}$ et à une déshydratation hyponatrémique dans une faible proportion de patients. 
La grande majorité des hommes mucoviscidosiques (98\%) est infertile en raison d'anomalies du développement de structures dérivant du canal de Wolff. Il s'agit notamment d'une malformation ou d'une absence des canaux déférents et d'anomalies des vésicules séminales. Des anomalies de la motilité et de la morphologie du sperme ont été également décrites, bien que du sperme prélevé par aspiration testiculaire ait permis des grossesses menées à terme.

\section{Contribution du génotype de CFTR}

Le gène CFTR code pour une protéine de 1480 acides aminés qui fonctionne comme un canal chlorure régulé par l'AMPc et comme un régulateur d'autres canaux ioniques. Le gène CFTR est responsable de la régulation du transport de liquides et d'ions à travers les membranes apicales des cellules épithéliales et joue un rôle central dans l'absorption et la sécrétion dans l'épithélium des voies aériennes et des canaux pancréatiques. Sa dysfonction induit des modifications de la teneur en eau et en $\mathrm{NaCl}$ du liquide de surface des voies aériennes et des sécrétions pancréatiques, ce qui aboutit à une altération des mécanismes de defense et à des obstructions qui détruisent ces deux organes par la suite. Plus de 1400 mutations du gène CFTR ont été rapportées au CF Genetic Analysis Consortium (www.genet.sickkids.on.ca/cftr); 1200 d'entre elles ont été associées à la maladie, et les 200 restantes paraissent être des variants bénins. Malgré cette extrême diversité allélique, une seule mutation, qui est une délétion de la phénylalanine à la position 508 (appelée $\Delta \mathrm{F} 508$ ), représente $70 \%$ des allèles mucoviscidosiques au plan mondial [10]. La mutation $\Delta$ F508 s'observe presque exclusivement chez des Caucasiens européens et sa fréquence s'accroît en remontant du bassin méditerranéen vers les parties nord de l'Europe [10]. Environ 5 à 20 autres mutations de CFTR représentent 10 à $20 \%$ des allèles mucoviscidosiques [10]. La répartition des mutations «moins fréquentes» varie selon les populations. Les mutations restantes de CFTR sont rares, à l'exception d'un très petit nombre, qui a atteint une fréquence élevée dans des populations isolées en raison d'un effet fondateur. La fréquence élevée de la mutation $\Delta$ F508 explique que le même génotype de CFTR $(\Delta \mathrm{F} 508 / \Delta \mathrm{F} 508)$ est présent chez $50 \%$ des patients mucoviscidosiques. Les homozygotes $\Delta$ F508 ont été étudiés de façon approfondie, et ce génotype est presque toujours associé à une insuffisance pancréatique [11]. La concentration de chlorure est élevée dans la sueur de ces sujets, et tous ceux de sexe masculin

Causes des variations phénotypiques de la mucoviscidose présentant ce génotype sont stériles. La sévérité de l'atteinte respiratoire varie cependant considérablement selon les homozygotes $\Delta$ F508 $[11,12]$.

L'important groupe des patients mucoviscidosique dont le génotype de CFTR est identique (homozygotie $\Delta$ F508) constitue une population de référence pour l'évaluation de l'effet d'autres mutations de ce gène sur le phénotype de la mucoviscidose. Des études des relations génotype/phénotype ont révélé que le génotype de CFTR était étroitement corrélé à la sévérité de l'atteinte pancréatique [11]. La plupart des mutations sont associées à une insuffisance pancréatique, mais environ 24 le sont à une suffisance pancréatique. Ces mutations altèrent la fonction du gène CFTR mais ne l'abolissent pas [13]. De ce fait, une fonction pancréatique résiduelle paraît résulter d'un fonctionnement partiel de ce gène. Cette association n'est cependant pas absolue; certains patients porteurs de mutations autorisant un certain fonctionnement du gène CFTR sont atteints d'une insuffisance pancréatique [13]. Certaines mutations produisent également des anomalies spécifiques de la fonction pancréatique, par exemple L99TF, qui est étroitement associée à une pancréatite [14]. Les complications digestives telles qu'un IM surviennent généralement chez des patients porteurs de mutations induisant une insuffisance pancréatique. Le taux d'IM est toutefois plus faible chez les patients porteurs de la mutation G551D, qui provoque une insuffisance pancréatique, que chez les homozygotes $\Delta \mathrm{F} 508[15,16]$. La suffisance pancréatique a été associée à des troubles respiratoires moins sévères, mais il a été difficile de déterminer des mutations spécifiques induisant à la fois une suffisance pancréatique et une atteinte respiratoire discrète. Un exemple en est la mutation A455E. Deux études ont montré que la progression de l'atteinte respiratoire était plus lente chez les patients porteurs de cette mutation que chez ceux homozygotes pour la mutation $\Delta$ F508 $[17,18]$.

Le génotype de CFTR est faiblement corrélé au degré d'anomalie de la concentration de chlorure dans la sueur, et ceci survient généralement dans le contexte d'une suffisance pancréatique. En d'autres termes, les patients porteurs de mutations autorisant une fonction partielle permettant une suffisance pancréatique tendent également à présenter des élévations moins anormales de cette concentration [19]. Enfin, les anomalies des voies génitales masculines dues à la mucoviscidose sont étroitement associées au génotype de CFTR. La mutation de site d'épissage $3849+10 \mathrm{kbC} \rightarrow \mathrm{T}$ est associée à un taux élevé de fertilité chez les hommes atteints d'une mucoviscidose, bien qu'elle induise des troubles respiratoires de type mucoviscidosique [20]. 
Deux mutations de CFTR démontrent à quel point cette association entre génotype et phénotype peut être subtile. Une mutation responsable de mucoviscidose $(\mathrm{R} 117 \mathrm{H})$ est également fréquente chez les hommes présentant de façon isolée une absence bilatérale congénitale des canaux déférents $(\mathrm{ABCD})$. Cette dernière situation possède des caractéristiques identiques à celles observées chez les sujets de sexe masculin présentant une mucoviscidose, mais les hommes atteints d'une ABCD ne présentent pas d'atteinte respiratoire réduisant l'espérance de vie, bien que pouvant être porteurs de mutations de chaque gène CFTR. La différence entre les hommes atteints d'une mucoviscidose et ceux présentant une $\mathrm{ABCD}$, qui sont tous porteurs de la mutation $\mathrm{R} 117 \mathrm{H}$, est qu'une seconde mutation est présente dans le gène de ceux du premier de ces deux groupes [21]. Ainsi, la présence d'une seconde altération (appelée 5T) réduisant la quantité de protéine CFTR produite induit également la survenue d'une atteinte respiratoire réduisant l'espérance de vie chez les hommes présentant une ABCD. Il est intriguant de constater que la mutation $5 \mathrm{~T}$ induit par elle-même une mucoviscidose quant elle est associée à une anomalie de la longueur d'un variant adjacent de séquence appelé séquence TG. Les hommes porteurs d'une séquence TG plus longue associée au variant $5 \mathrm{~T}$ et d'une autre mutation du gène CFTR (telle que $\Delta \mathrm{F} 508$ ) sont généralement atteints d'une $\mathrm{ABCD}$. Ceux porteurs de la même association de mutations ( $\Delta$ F508 et $5 \mathrm{~T})$ et de la séquence TG courte plus fréquente sont généralement exempts d'anomalie [22]. En raison de cette association entre le génotype de CFTR et le phénotype mucoviscidosique, les multiples altérations de CFTR doivent être considérées comme contribuant à l'évolution clinique.

\section{Contributions de modificateurs génétiques}

Certaines données sont en faveur d'une association entre des mutations induisant une mucoviscidose et l'atteinte respiratoire (par exemple A455E et $\mathrm{R} 117 \mathrm{H}$, comme décrit plus haut), mais le génotype de CFTR ne permet pas le plus souvent de prévoir la sévérité des troubles respiratoires. Cette situation est principalement due à l'importante variabilité de l'état de la fonction respiratoire observée chez les patients mucoviscidosiques dont le génotype de CFTR est identique [12]. Cette observation indique que des facteurs indépendants de CFTR doivent substantiellement contribuer aux variations de l'atteinte respiratoire.
L'une des méthodes les plus efficientes de détermination du rôle de facteurs génétiques dans la variabilité d'une maladie chez l'homme consiste à étudier des familles dont plusieurs enfants sont atteints. Les frères et sœurs ont $50 \%$ de gènes en commun. De ce fait, la récidive de complications dans une fratrie à un taux plus élevé que chez des patients non apparentée peut impliquer des facteurs génétiques. Un exemple en est l'IM. Si le premier-né d'une famille est atteint d'une mucoviscidose et d'un IM, chaque enfant suivant atteint d'une mucoviscidose sera exposé à un risque de $25 \%$ de l'être également d'un IM. Le risque de récidive dans une fratrie est substantiellement plus élevé que chez des patients non apparentés (environ 15\%). D’autres exemples de complications avec risque élevé de récidive et présentant des caractéristiques pathologiques hautement similaires entre les membres d'une fratrie sont les troubles de la fonction pancréatique, l'atteinte hépatique et les infections à Pseudomonas [23-26]. Les membres d'une fratrie partagent toutefois le même environnement (domicile, école, établissement de soins, etc.) et, de ce fait, des taux plus élevés de récidive chez ces sujets n'impliquent pas nécessairement des modificateurs génétiques. Un test formel de l'effet d'un modificateur génétique par rapport à celui d'un modificateur non génétique consiste à étudier des jumeaux atteints. La comparaison de jumeaux monozygotes (100\% de gènes en commun) et dizygotes ( $50 \%$ de gènes en commun) permet d'estimer les effets génétiques (héritabilité). En utilisant cette approche, des investigateurs ont montré qu'une mesure de la masse corporelle (la taille pour le poids) était principalement déterminée par des gènes [27]. Des facteurs génétiques indépendants du CFTR paraissent également influencer la fonction intestinale chez les patients mucoviscidosiques en altérant la sécrétion de chlorure $[28,29]$ et peuvent modifier la croissance de ces sujets [30].

Une méthode d'identification de gènes modifiant spécifiquement le phénotype mucoviscidosique consiste à sélectionner des gènes candidats sur la base des connaissances de la physiopathologie de la mucoviscidose. Ces gènes candidats codent pour des protéines qui jouent un rôle dans la progression de la maladie (par exemple des médiateurs de l'inflammation). Des variants ADN sont identifiés dans ces gènes candidats et leur répartition est déterminée chez des patients mucoviscidosiques stratifiés en fonction de la sévérité de la maladie. Si les résultats indiquent que les variants d'un gène candidat sont associés à une plus grande sévérité de la maladie, il est possible de conclure que ce candidat est un modificateur. Cette approche est puissante, mais est cependant enta- 
chée du problème inhérent à la répartition des patients en différents groupes. Des variants génétiques peuvent différer de façon fortuite ou en raison de différences non reconnues entre les deux groupes, ce qui affecte la répartition des variants qui ne sont pas corrélés à la sévérité de la maladie. De ce fait, les études d'association cas-témoins tendent à identifier un certain nombre de gènes modificateurs candidats faussement positifs. Une approche destinée à réduire ces faux positifs est de tester des gènes candidats dans différentes populations de patients. Drumm et coll. [31] ont testé selon cette approche dix gènes candidats dont des études précédente avaient montré qu'ils modifiaient la sévérité d'une mucoviscidose. Leur étude multicentrique a été menée chez 808 patients mucoviscidosiques recrutés sur la base de la sévérité de la maladie. Sur ces dix variants génétiques, un seul gène «modificateur» (celui du facteur transformant la croissance $\beta 1$, TGF $\beta 1$ ) a été associé à une atteinte respiratoire plus sévère. Les auteurs ont confirmé leurs résultats en testant le gène du TGF $\beta 1$ dans un second groupe de 498 patients atteints d'une mucoviscidose [31]. Des variants de ce gène ont été associés à une sensibilité à l'asthme et à une protection contre la survenue d'une bronchopneumopathie chronique obstructive chez des fumeurs, ce qui a conforté le concept selon lequel des gènes modificateurs intervenant dans la mucoviscidose peuvent également jouer un rôle pathologique dans des affections plus banales [32-35]. Des variants de deux autres gènes (ceux du facteur de nécrose tumorale $\alpha$ et de la lectine liant le mannose) ont été associés à la sévérité de l'atteinte respiratoire indépendamment du génotype de CFTR dans plus d'un groupe de patients mucoviscidosiques [36-40]. Drumm et coll. [31] ont inclus ces deux gènes dans une étude de reproductibilité et n'ont pas observé d'association avec la sévérité de l'atteinte respiratoire. Les gènes modificateurs dont l'effet est réel mais discret ne peuvent cependant pas être détectés par toute étude. Des effets modificateurs subtils sur le phénotype peuvent être masqués par des variations dues à des facteurs non génétiques tels qu'une différence entre les traitements effectués par les divers centres [41]. Il n'est donc pas surprenant que divers autres gènes modificateurs candidats n'aient été associés à la sévérité de la maladie qu'une fois ou n'aient pas été constamment associés à celle-ci [42].

Dans les années qui viennent, il est probable que de nouvelles études confirmeront ou réfuteront le rôle d'un certain nombre de gènes dans la modification du phénotype de la mucoviscidose. De plus, il est prévisible que d'autres approches de l'identification de gènes modificateurs telles que le clonage positionnel révèleront des gè-

Causes des variations phénotypiques de la mucoviscidose nes qui n'auraient pas été sélectionnés par la méthode des gènes candidats. Ces gènes pourraient posséder des fonctions différentes de celles envisagées pour les modificateurs, mais, en raison de leur corrélation génétique avec la maladie, il peut être démontré qu'ils sont des modificateurs de la mucoviscidose. Cette approche a été appliquée afin d'identifier le CFTR comme gène responsable de la mucoviscidose. Les études de familles sont idéalement adaptées à la dernière approche. Les membres des fratries atteints d'une mucoviscidose peuvent être regroupés en fonction du degré de similitude afin de mesurer la sévérité de la maladie (par exemple atteinte respiratoire). Ces sujets sont alors testés à la recherche de corrélations entre le degré de partage d'une région génétique et le degré de similitude de la sévérité de la maladie. Il est ainsi possible de tester deux membres d'une fratrie présentant une atteinte respiratoire sévère afin de recherchez des gènes modificateurs communs responsables de celle-ci. Des frères ou sœurs différents, l'un sévèrement atteint, l'autre légèrement, ne devraient pas partager un même gène modificateur. Cette méthode, appelée concordance, a été appliqués à des membres d'une fratrie atteints d'une mucoviscidose afin de démontrer un rôle d'une région du chromosome 19 qui pourrait contenir un modificateur ayant trait à l'IM [43]. Une puissante extension des méthodes basées sur des familles consiste à tester des parents et leurs enfants mucoviscidosiques afin d'identifier une transmission de gènes modificateurs. Dans cette situation, l'objectif est de déterminer si un variant d'un gène modificateur a été transmis plus souvent que prévu sur la base du hasard à des enfants regroupés en fonction de la sévérité de la maladie. Cette méthode, appelée test de déséquilibre de la transmission (TDT), n'est pas sujette aux erreurs dues à une stratification, qui sont le fléau des études d'association. Il est toutefois nécessaire de tester les deux patients afin que l'efficacité du TDT soit maximale. L'obtention de l'ADN de tous les membres de la famille peut être difficile, notamment quand les patients sont âgés. Les études basées sur des familles menées en Europe, au Canada et aux ÉtatsUnis disposent cependant d'ensemble de patients adéquats pour un TDT.

\section{Rôle de modificateurs environnementaux}

L'amélioration de la survie des patients mucoviscidosiques au cours des cinq ou six dernières décennies ne peut être attribuée à des gènes modificateurs. Les modifications du traitement, particulièrement le développe- 
Fig. 1. Causes de variation des principales manifestations de la mucoviscidose. La contribution relative du génotype de CFTR, des gènes modificateurs et des facteurs environnementaux est estimée pour chacun des appareils et organes affectés par la mucoviscidose. Le génotype de CFTR est le principal déterminant de la sévérité de l'atteinte pancréatique et des malformations de l'appareil génital masculin. Des gènes modificateurs et des facteurs environnementaux jouent un rôle important dans la sévérité de l'atteinte respiratoire.

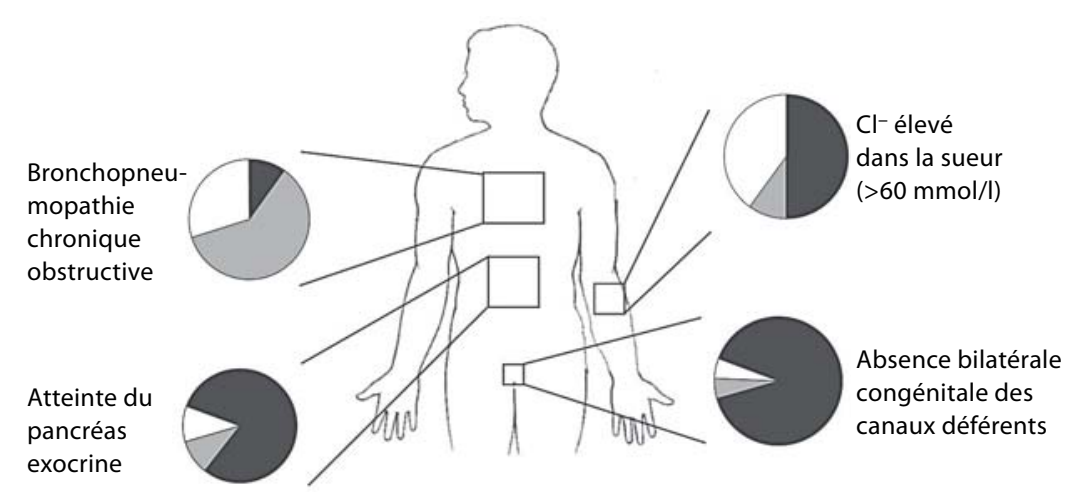

Génotype de CFTR
Facteurs environnementaux ment du traitement substitutif par enzymes pancréatiques et la création de centres multidisciplinaires de prise en charge de la mucoviscidose, ont nettement modifié l'évolution de cette maladie (voir «Soins des patients atteints d'une mucoviscidose» par B. Strandvik dans ce même numéro). Ces «facteurs modifiants» sont de nature environnementale. L'identification d'expositions environnementales spécifiques influençant l'évolution clinique est une méthode puissante de détermination de facteurs accessibles à une intervention. L'évaluation de la contribution de l'environnement au phénotype a été difficile en raison de la diversité des composantes impliquées et de l'absence de mesure objective pour un grand nombre d'entre elles. Malgré ces limites, le tabagisme passif a été relié à une altération de la fonction respiratoire [44-46]. La suppression de l'exposition des patients mucoviscidosiques à la fumée de tabac est une mesure relativement simple pour améliorer l'évolution. Des études ont montré qu'une intervention nutritionnelle intensive modifiait également l'évolution [47-50]. Il est intéressant de noter que la réponse des patients au traitement nutritionnel est variable, ce qui suggère que d'autres facteurs environnementaux et/ou génétiques peuvent intervenir [51]. Une colonisation des voies respiratoires par P. aeruginosa et Burkholderia cepacia est un événement lié à l'environnement $[52,53]$ et est associée à un réduction de la longévité [54-57]. Ici encore, des modifications de facteurs environnementaux, tels que le contrôle des infections, peuvent prévenir la dissémination de soustypes virulents de ces bactéries.
L'amélioration de l'évolution clinique par la réduction des variations de traitement entre centres cliniques afin que tous les patients reçoivent des soins optimisés est un des piliers de la prise en charge actuelle de la mucoviscidose. Des fondations dédiées à la défense des patients ont utilisé leurs ressources et aptitudes organisationnelle afin d'établir des centres de soins multidisciplinaires qui permettent d'optimiser et d'uniformiser le traitement. Aux États-Unis, de récents efforts ont visé à améliorer le statut nutritionnel des patients mucoviscidosiques, sachant qu'une malnutrition est associée à une atteinte respiratoire plus grave et à une évolution clinique plus sévère. Enfin, l'observance est manifestement un point clé pour les patients mucoviscidosiques, comme dans toute affection chronique. Même si un traitement modifiant la fonction de la protéine CFTR pouvait être développé, sa réussite devrait dépendre de la volonté du patient d'adhérer à vie à un schéma thérapeutique. De ce fait, l'accent mis sur les comportements sains et l'observance des soins de routine et d'une prise en charge anticipatrice seront probablement à l'origine de la poursuite de l'amélioration de l'évolution clinique de la mucoviscidose.

\section{Conclusion}

Le traitement de la mucoviscidose a connu des succès étonnants, alors que cette maladie était jadis fréquemment fatale au cours de la première année de la vie. L’identification des facteurs clés qui déterminent l'évolution 
clinique chez chaque patient et le développement de traitements destinés à leur répondre accroissent la longévité de tous les sujets atteints d'une mucoviscidose. L'identification du gène du CFTR a été une étape majeure du progrès des connaissances de la physiopathologie à l'échelon moléculaire et de la détermination du degré auquel la fonction du CFTR influence l'évolution d'une mucovis- cidose. Ces études ont révélé que d'autres facteurs modificateurs, tant génétiques que non génétiques, jouaient un rôle important dans la détermination de la sévérité de cette maladie (fig. 1). Pour la prochaine décennie, le défi sera d'identifier et de caractériser ces facteurs afin d'optimiser l'évolution clinique chez tous les patients atteints d'une mucoviscidose.

\section{Références}

1 Cutting GR: Cystic fibrosis; in Emery and Rimoin's Principles and Practice of Medical Genetics, ed 4. London, Churchill-Livingston, 2002.

- Fujiwara TM, Morgan K, Schwartz RH, et al: Genealogical analysis of cystic fibrosis families and chromosome 7q RFLP haplotypes in the Hutterite Brethren. Am J Hum Genet 1989;44:327-337.

-3 Super M: Cystic fibrosis in the South West African Afrikaner. An example of population drift, possibly with heterozygote advantage. S Afr Med J 1975;49:818-820.

4 Cystic Fibrosis Foundation: Cystic Fibrosis Foundation Patient Registry Annual Data Report 1999. Bethesda, Cystic Fibrosis Foundation, 2000.

5 Gaskin KJ, Gurwitz D, Durie P, et al: Improved respiratory prognosis in patients with cystic fibrosis with normal fat absorption. J Pediatr 1982;100:857-862.

6 Cystic Fibrosis Foundation: Cystic Fibrosis Foundation Patient Registry Annual Data Report 2004. Bethesda, Cystic Fibrosis Foundation, 2004.

7 Marshall BC, Butler SM, Stoddard M, et al: Epidemiology of cystic fibrosis-related diabetes. J Pediatr 2005;146:681-687.

8 di Sant'Agnese PA, Hubbard VA: The hepatobiliary system; in Taussig LM (ed): Cystic Fibrosis. New York, Thieme Stratton, 1984 pp 296-322.

9 Dray X, Bienvenu T, Desmazes-Dufeu N, et al: Distal intestinal obstruction syndrome in adults with cystic fibrosis. Clin Gastroenterol Hepatol 2004;2:498-503.

10 Bobadilla JL, Macek M, Fine JP, Farrell PM: Cystic fibrosis: a worldwide analysis of CFTR mutations - correlation with incidence data and application to screening. Hum Mutat 2002;19:575-606.

$\checkmark 11$ Kerem E, Corey M, Kerem B-S, et al: The relation between genotype and phenotype in cystic fibrosis-analysis of the most common mutation (deltaF508). N Engl J Med 1990; 323:1517-1522.

12 Cystic Fibrosis Genotype-Phenotype Consortium: Correlation between Genotype and Phenotype in Patients with Cystic Fibrosis. N Engl J Med 1993;329:1308-1313.
13 Koch C, Cuppens H, Rainisio M, et al: European Epidemiologic Registry of Cystic Fibrosis (ERCF): comparison of major disease manifestations between patients with different classes of mutations. Pediatr Pulmonol 2001;31:1-12.

14 Gomez LM, Benetazzo MG, Marzari MG, et al: High frequency of cystic fibrosis transmembrane regulator mutation L997F in patients with recurrent idiopathic pancreatitis and in newborns with hypertrypsinemia Am J Hum Genet 2000;66:2013-2014.

15 Hamosh A, King TM, Rosenstein BJ, et al: Cystic fibrosis patients bearing the common missense mutation Gly $\rightarrow$ Asp at codon 551 and the deltaF508 are indistinguishable from deltaF508 homozygotes except for decreased risk of meconium ileus. Am J Hum Genet 1992;51:245-250.

16 Feingold J, Guilloud-Bataille M: Genetic comparisons of patients with cystic fibrosis with or without meconium ileus. Clinical Centers of the French CF Registry. Ann Genet 1999;42:147-150.

-17 Gan KH, Veeze HJ, van den Ouweland AMW, et al: A cystic fibrosis mutation associated with mild lung disease. N Engl J Med 1995;333:95-99.

18 De Braekeleer M, Allard C, Leblanc J-P, et al: Genotype-phenotype correlation in cystic fibrosis patients compound heterozygous for the A455E mutation. Hum Genet 1997;101: 208-211.

19 Wilschanski M, Zielenski J, Markiewicz D, et al: Correlation of sweat chloride concentration with classes of the cystic fibrosis transmembrane conductance regulator gene mutations. J Pediatr 1995;127:705-710.

20 Highsmith WE Jr, Burch LH, Zhou Z, et al: A novel mutation in the cystic fibrosis gene in patients with pulmonary disease but normal sweat chloride concentrations. N Engl J Med 1994;331:974-980.

21 Kiesewetter S, Macek M Jr, Davis C, et al: A mutation in the cystic fibrosis transmembrane conductance regulator gene produces different phenotypes depending on chromosomal background. Nat Genet 1993;5:274278.
22 Groman JD, Hefferon TW, Casals T, et al: Variation in a repeat sequence determines whether a common variant of the cystic fibrosis transmembrane conductance regulator gene is pathogenic or benign. Am J Hum Genet 2004;74:176-179.

23 Corey M, Durie P, Moore D, et al: Familial concordance of pancreatic function in cystic fibrosis. J Pediatr 1989;115:274-277.

24 Santis G, Osborne L, Knight RA, Hodson ME: Independent genetic determinants of pancreatic and pulmonary status in cystic fibrosis. Lancet 1990;336:1081-1084.

25 Duthie A, Doherty DG, Williams C, et al: Genotype analysis for deltaF508, G551D and R553X mutations in children and young adults with cystic fibrosis with and without liver disease. Hepatology 1992;15:660-664.

26 Picard E, Aviram M, Yahav Y, et al: Familial concordance of phenotype and microbial variation among siblings with CF. Pediatr Pulmonol 2004;38:292-297.

27 Mekus F, Ballmann M, Bronsveld I, et al: Categories of deltaF508 homozygous cystic fibrosis twin and sibling pairs with distinct phenotypic characteristics. Twin Res 2000;3: 277-293.

28 Bronsveld I, Mekus F, Bijman J, et al: Residual chloride secretion in intestinal tissue of deltaF508 homozygous twins and siblings with cystic fibrosis. The European CF Twin and Sibling Study Consortium. Gastroenterology 2000;119:32-40.

29 Bronsveld I, Mekus F, Bijman J, et al: Chloride conductance and genetic background modulate the cystic fibrosis phenotype of Delta F508 homozygous twins and siblings. J Clin Invest 2001;108:1705-1715

- 30 Mekus F, Laabs U, Veeze H, Tummler B: Genes in the vicinity of CFTR modulate the cystic fibrosis phenotype in highly concordant or discordant F508del homozygous sib pairs. Hum Genet 2003;112:1-11.

31 Drumm ML, Konstan MW, Schluchter MD, et al: Genetic modifiers of lung disease in cystic fibrosis. N Engl J Med 2005;353:14431453.

32 Pulleyn LJ, Newton R, Adcock IM, Barnes PJ: TGFbetal allele association with asthma severity. Hum Genet 2001;109:623-627. 
>33 Wu L, Chau J, Young RP, Pokorny V, et al: Transforming growth factor-betal genotype and susceptibility to chronic obstructive pulmonary disease. Thorax 2004;59:126129.

34 Silverman ES, Palmer LJ, Subramaniam V, et al: Transforming growth factor-betal promoter polymorphism C-509T is associated with asthma. Am J Respir Crit Care Med 2004;169:214-219.

$>35$ Celedon JC, Lange C, Raby BA, et al: The transforming growth factor-betal (TGFB1) gene is associated with chronic obstructive pulmonary disease (COPD). Hum Mol Genet 2004;13:1649-1656.

36 Hull J, Thomson AH: Contribution of genetic factors other than CFTR to disease severity in cystic fibrosis. Thorax 1998;53:10181021.

\$37 Davies JC, Turner MW, Klein N: Impaired pulmonary status in cystic fibrosis adults with two mutated MBL-2 alleles. Eur Respir J 2004;24:798-804.

38 Yarden J, Radojkovic D, De Boeck K, et al: Association of tumour necrosis factor alpha variants with the CF pulmonary phenotype. Thorax 2005;60:320-325.

39 Garred P, Pressler T, Madsen HO, et al: Association of mannose-binding lectin gene heterogeneity with severity of lung disease and survival in cystic fibrosis. J Clin Invest 1999;104:431-437.

-40 Arkwright PD, Laurie S, Super M, et al: TGFbeta(1) genotype and accelerated decline in lung function of patients with cystic fibrosis. Thorax 2000;55:459-462.
41 Davies JC, Griesenbach U, Alton E: Modifier genes in cystic fibrosis. Pediatr Pulmonol 2005;39:383-391.

42 Cutting GR: Modifier genetics: cystic fibrosis. Annu Rev Genomics Hum Genet 2005;6: 237-260.

43 Zielenski J, Corey M, Rozmahel R, et al: Detection of a cystic fibrosis modifier locus for meconium ileus on human chromosome 19q13. Nat Genet 1999;22:128-129.

44 Rubin BK: Exposure of children with cystic fibrosis to environmental tobacco smoke. N Engl J Med 1990;323:782-788.

45 Kovesi T, Corey M, Levison H: Passive smoking and lung function in cystic fibrosis. Am Rev Respir Dis 1993;148:1266-1271.

46 Smyth A, O’Hea U, Williams G, et al: Passive smoking and impaired lung function in cystic fibrosis. Arch Dis Child 1994;71:353354.

47 Shepherd R, Cooksley WG, Cooke WD: Improved growth and clinical, nutritional, and respiratory changes in response to nutritional therapy in cystic fibrosis. J Pediatr 1980 97:351-357.

48 Dalzell AM, Shepherd RW, Dean B, et al: Nutritional rehabilitation in cystic fibrosis: a 5 year follow-up study. J Pediatr Gastroenterol Nutr 1992;15:141-145.

49 Steinkamp G, von der Hardt H: Improvement of nutritional status and lung function after long-term nocturnal gastrostomy feedings in cystic fibrosis. J Pediatr 1994;124: 244-249.
50 Zemel BS, Jawad AF, Fitzsimmons S, Stallings VA: Longitudinal relationship among growth, nutritional status, and pulmonary function in children with cystic fibrosis: analysis of the cystic fibrosis foundation national CF patient registry. J Pediatr 2000;137: 374-380.

51 Kirvela O, Stern RC, Askanazi J, et al: Longterm parenteral nutrition in cystic fibrosis. Nutrition 1993;9:119-126.

52 John M, Ecclestone E, Hunter E, et al: Epidemiology of Pseudomonas cepacia colonization among patients with cystic fibrosis. $\mathrm{Pe}$ diatr Pulmonol 1994;18:108-113.

53 Farrell PM, Shen G, Splaingard M, et al: Acquisition of Pseudomonas aeruginosa in children with cystic fibrosis. Pediatrics 1997; 100:E2.

54 Kerem E, Corey M, Gold R, Levison H: Pulmonary function and clinical course in patients with $\mathrm{CF}$ after pulmonary colonization with Pseudomonas aeruginosa. J Pediatr 1990;116:714-719.

55 Demko CA, Byard PJ, Davis PB: Gender differences in cystic fibrosis: Pseudomonas aeruginosa infection. J Clin Epidemiol 1995;48: 1041-1049.

56 Corey M, Farewell V: Determinants of mortality from cystic fibrosis in Canada, 19701989. Am J Epidemiol 1996;143:10071017.

57 Parad RB, Gerard CJ, Zurakowski D, et al: Pulmonary outcome in cystic fibrosis is influenced primarily by mucoid Pseudomonas aeruginosa infection and immune status and only modestly by genotype. Infect Immun 1999;67:4744-4750. 